\title{
Schwinger Pair Production in Electric and Magnetic Fields
}

\author{
Sang Pyo Kin* \\ Department of Physics, Kunsan National University, Kunsan 573-701, Korea and \\ Asia Pacific Center for Theoretical Physics, Pohang 790-784, Korea
}

Don N. Pagषi

Theoretical Physics Institute, Department of Physics, University of Alberta, Edmonton, Alberta, Canada T6G 2J1

(Dated: February 1, 2008)

\begin{abstract}
Charged particles in static electric and magnetic fields have Landau levels and tunneling states from the vacuum. Using the instanton method of Phys. Rev. D 65, 105002 (2002), we obtain the formulae for the pair-production rate in spinor and scalar QED, which sum over all Landau levels and recover exactly the well-known results. The pair-production rates are calculated for an electric field of finite extent, and for the Sauter potential, both with a constant magnetic field also present, and are shown to have finite-size effects.
\end{abstract}

PACS numbers: PACS number(s): 12.20.-m, 13.40.-f

\section{INTRODUCTION}

Vacuum polarization and pair production are two physically important phenomena of quantum electrodynamics (QED) in strong electromagnetic fields [1, 2]. The one-loop effective action in constant electric and magnetic fields has nonlinear contributions to the classical action and, thereby, leads to the nonlinear Maxwell equations. Another interesting phenomenon is the pair production due to vacuum instability in the presence of electric fields near and above the critical electric field strength $E_{c}=m^{2} c^{3} / e \hbar\left(1.3 \times 10^{16} \mathrm{~V} / \mathrm{cm}\right)[1,2]$ (for references, see, e.g., [3, 44)).

Strong QED has many physical applications [5]. In particular, electromagnetic fields of some neutron stars and black holes [6] and high-intensity laser fields [7] above the critical strength $E_{c} / c=B_{c}=m^{2} c^{2} / e \hbar\left(4.4 \times 10^{13} G\right)$ have revived recent interest and applications of strong QED. The one-loop QED effective action is known exactly in the background of constant electric and magnetic fields [2]. In the case in which $\mathbf{E}^{2}-\mathbf{B}^{2}$ and $\mathbf{E} \cdot \mathbf{B}$ are not both zero, one can go to a frame in which $\mathbf{E}$ and $\mathbf{B}$ are parallel with magnitude $E$ and $B$, and obtain the imaginary part of the one-loop effective action per four-volume for spinor QED given by [1, 2, 8,9

$$
2 \operatorname{Im} \mathcal{L}_{\text {spinor }}^{(1)}=w_{\text {spinor }}^{(1)}=\frac{(q E)(q B)}{(2 \pi)^{2}} \sum_{n=1}^{\infty} \frac{1}{n} \operatorname{coth}\left(\frac{n \pi B}{E}\right) \exp \left(-\frac{n \pi m^{2}}{q E}\right),
$$

and that for scalar QED by 1, 10, 11, 12.

$$
2 \operatorname{Im} \mathcal{L}_{\text {scalar }}^{(1)}=w_{\text {scalar }}^{(1)}=\frac{(q E)(q B)}{2(2 \pi)^{2}} \sum_{n=1}^{\infty} \frac{(-1)^{n+1}}{n} \operatorname{csch}\left(\frac{n \pi B}{E}\right) \exp \left(-\frac{n \pi m^{2}}{q E}\right) .
$$

The pair-production rates per volume per time themselves are the first terms in each of these series [13],

$$
\mathcal{N}_{\text {spinor }}^{(1)}=\frac{(q E)(q B)}{(2 \pi)^{2}} \operatorname{coth}\left(\frac{\pi B}{E}\right) \exp \left(-\frac{\pi m^{2}}{q E}\right)
$$

and

$$
\mathcal{N}_{\text {scalar }}^{(1)}=\frac{(q E)(q B)}{2(2 \pi)^{2}} \operatorname{csch}\left(\frac{\pi B}{E}\right) \exp \left(-\frac{\pi m^{2}}{q E}\right)
$$

Kruglov derived a general formula for the more general case of particles of arbitrary spin $s$ with ADM (anomalous magnetic moment) and EDM (electric dipole moment) [14].

\footnotetext{
*Electronic address: sangkim@kunsan.ac.kr;spkim@phys.ualberta.ca
}

${ }^{\dagger}$ Electronic address: don@phys.ualberta.ca 
In this paper we apply the recently-introduced instanton method [3] to find the pair-production rate in constant electric and magnetic fields in spinor and scalar QED, agreeing with the results above. The idea of the instanton method, the elaboration of the role of tunneling in pair production [15], is that for static electric fields, fermions and bosons have tunneling states from the vacuum in the Coulomb (space-dependent) gauge, and their pair-production rates are determined by these instanton actions for tunneling. In the presence of static magnetic fields, charged fermions and bosons have discrete spectrum of Landau levels. Taking into account both Landau levels and instanton actions for tunneling, we obtain equivalent formulae for pair-production rates in spinor and scalar QED. These formulae are given as sums over all Landau levels, and we recover exactly the well-known results. Further, applying the method, we estimate the pair-production rates by an electric field with a finite extent and a constant magnetic field, which have additional factors determined by the potential difference across the boundary beside the pair-production rate for a constant electromagnetic field.

The organization of this paper is as follows. In Sec. II we generalize the instanton method to the case of constant electric and magnetic fields and obtain the pair-production rate as the sum of Landau levels. We also compare our instanton method with the instanton method in Euclidean time. In Sec. III we apply the instanton method to inhomogeneous electric fields: a constant electric field confined to a finite region and one from the Sauter-type potential.

\section{INSTANTON METHOD FOR PARTICLES WITH SPIN IN ELECTROMAGNETIC FIELDS}

With the spin properly taken into account, the component equation for minimally-coupled particles with spin in a constant electric field takes the form (in units with $\hbar=c=1$ and with metric signature $(+,-,-,-)$ )

$$
\left[\eta^{\mu \nu}\left(\partial_{\mu}+i q A_{\mu}\right)\left(\partial_{\nu}+i q A_{\nu}\right)+m^{2}+2 i \sigma q E\right] \Phi_{\sigma}=0
$$

where $q(q>0), m$ and $\sigma$ are the charge, mass and spin of the particles. We shall show the physical implication of the imaginary part from the spin effect. After mode-decomposition,

$$
\left[-\partial_{z}^{2}-(\omega+q E z)^{2}+m^{2}+\mathbf{k}_{\perp}^{2}+2 i \sigma q E\right] \Phi_{\sigma \omega \mathbf{k}_{\perp}}(z)=0 .
$$

The wave function (6) has a solution in terms of the complex parabolic cylindrical function $E(x, y)$ [3, 16],

$$
\Phi_{\sigma \omega \mathbf{k}_{\perp}}(z)=C E\left(\tilde{a}_{s \mathbf{k}_{\perp}}, \xi\right)
$$

where

$$
\xi=\sqrt{\frac{2}{q E_{0}}}(\omega+q E z), \quad \tilde{a}_{s \mathbf{k}_{\perp}}=\frac{m^{2}+\mathbf{k}_{\perp}^{2}}{2 q E}+i \sigma .
$$

In the two asymptotic regions the wave function becomes

$$
\begin{aligned}
& \Phi_{\sigma \omega \mathbf{k}_{\perp}}(z)=A \varphi_{\sigma \omega \mathbf{k}_{\perp}}(\xi)-B \varphi_{\sigma \omega \mathbf{k}_{\perp}}^{*}(\xi), \quad\left(\xi \ll-2\left|\tilde{a}_{s \mathbf{k}_{\perp}}\right|^{1 / 2}\right), \\
& \Phi_{\sigma \omega \mathbf{k}_{\perp}}(z)=C \varphi_{\sigma \omega \mathbf{k}_{\perp}}^{*}(\xi), \quad\left(\xi \gg 2\left|\tilde{a}_{s \mathbf{k}_{\perp}}\right|^{1 / 2}\right),
\end{aligned}
$$

where

$$
\varphi_{\sigma \omega \mathbf{k}_{\perp}}(\xi)=\sqrt{\frac{2}{|\xi|}} e^{-(i / 4) \xi^{2}}
$$

and

$$
A=i C\left(1+e^{2 \pi \tilde{a}_{s \mathbf{k}_{\perp}}}\right)^{1 / 2}, \quad B=-i C e^{\pi \tilde{a}_{s \mathbf{k}_{\perp}} .}
$$

For bosons $(\sigma=0,1,2, \cdots)$, we have the flux conservation

$$
|A|^{2}=|B|^{2}+|C|^{2} .
$$

The reflection probability is given by

$$
\left|\frac{B}{A}\right|^{2}=\frac{e^{\pi\left(\tilde{a}_{s \mathbf{k}_{\perp}}^{*}+\tilde{a}_{s \mathbf{k}_{\perp}}\right)}}{\left[\left(1+e^{2 \pi \tilde{a}_{s \mathbf{k}_{\perp}}^{*}}\right)\left(1+e^{2 \pi \tilde{a}_{s \mathbf{k}_{\perp}}}\right)\right]^{1 / 2}}=\frac{1}{1+e^{-2 S_{\mathbf{k}_{\perp}}}},
$$


where $S$ is the instanton action

$$
S_{\mathbf{k}_{\perp}}=\pi \frac{m^{2}+\mathbf{k}_{\perp}^{2}}{2 q E} .
$$

This is with the boundary condition that the current flux on the right hand side is outward. However, then the group velocity there is inward (to the left). If we instead impose the boundary condition from causality that signals are outgoing on the right, then on the left hand side the outgoing flux becomes $|A / B|^{2}=1+e^{-2 S}$ times the incoming flux, an amplification by the Klein-paradox. Then by the results of Nikishov [13], the pair-production rate is just this amplification factor minus 1 , or $e^{-2 S}$. It is interesting that the instanton approximation with positive action $S$ (exact for a uniform field) never gives more than 1 expected pair per mode, though there is no such restriction for a general electric field.

On the other hand, for fermions $(\sigma=1 / 2,3 / 2, \cdots)$ there is the Klein-paradox, and the flux conservation now becomes

$$
|A|^{2}+|C|^{2}=|B|^{2}
$$

This is also with outgoing flux but incoming signals on the right. Replacing this by outgoing signals on the right makes the outgoing flux on the left become $|A / B|^{2}=1-e^{-2 S}$ times the incident flux on the left. (There is no amplification factor larger than one in the fermion case with causality imposed, because of the Pauli exclusion principle, as Feynman once explained to one of us (DNP) while drawing diagrams and saying, "I'm supposed to be good at these diagrams.") Then by Nikishov's results [13], the pair-production rate is just one minus this reflection coefficient, again $e^{-2 S}$. This result confirms the use of the instanton method in this paper and $\underline{3}]$.

In the instanton method [3] , we use the Klein-Gordon equation

$$
\left[\eta^{\mu \nu}\left(\partial_{\mu}+i q A_{\mu}\right)\left(\partial_{\nu}+i q A_{\nu}\right)+m^{2}\right] \Phi(t, \mathbf{x})=0
$$

The significant difference between Eqs. (5) and (16) is the imaginary constant term. We illustrate the instanton method by first considering the case of a pure electric field along the $z$ direction. In the Coulomb gauge, the 4 potential is given by

$$
A_{\mu}=(-E z, 0,0,0)
$$

The component field of the Klein-Gordon equation,

$$
\left[\left(\partial_{t}-i q E z\right)^{2}-\partial_{x}^{2}-\partial_{y}^{2}-\partial_{z}^{2}+m^{2}\right] \Phi=0
$$

has a solution of the form

$$
\Phi=e^{i\left(\mathbf{k}_{\perp} \cdot \mathbf{x}_{\perp}-\omega t\right)} \Phi_{\omega \mathbf{k}_{\perp}}(z)
$$

where $\mathbf{k}_{\perp}$ and $\mathbf{x}_{\perp}$ denote the momentum and the vector perpendicular to the electric field. Then the above equation becomes

$$
\left[-\partial_{z}^{2}-(\omega+q E z)^{2}+m^{2}+\mathbf{k}_{\perp}^{2}\right] \Phi_{\omega \mathbf{k}_{\perp}}(z)=0 .
$$

Note that Eq. (20) describes the wave equation for an inverted harmonic potential with a negative energy. Therefore, one has tunneling states under the potential barrier, whose instanton actions are given by [3]

$$
2 S_{\mathbf{k}_{\perp}}=\oint Q^{1 / 2}(z)=\pi \frac{m^{2}+\mathbf{k}_{\perp}^{2}}{q E},
$$

where $Q(z)=m^{2}+\mathbf{k}_{\perp}^{2}-(\omega+q E z)^{2}$. The tunneling state and instanton action can also be understood in classical theory. The mass of the charged particle is invariant:

$$
m^{2}=\eta^{\mu \nu}\left(p_{\mu}-q A_{\mu}\right)\left(p_{\nu}-q A_{\nu}\right)=\left(p_{0}+q E z\right)^{2}-p_{z}^{2}-\mathbf{p}_{\perp}^{2}
$$

The time component and the transverse component of 4-momentum are constants of motion, say, $p_{0}=\omega$ and $\mathbf{p}_{\perp}=\mathbf{k}_{\perp}$. In the Euclidean spacetime $t=i \tau$, the $z$ component becomes $p_{z}=i p_{z}^{E}$, where $p_{z}^{E}$ is the Euclidean momentum. Hence Eq. (22) becomes a harmonic oscillator in the reduced phase space $\left(z, p_{z}\right)$,

$$
\frac{1}{2} p_{z}^{2}+\frac{(q E)^{2}}{2}\left(z+\frac{\omega}{q E}\right)^{2}=\frac{1}{2}\left(m^{2}+\mathbf{k}_{\perp}^{2}\right) .
$$


The action of the oscillator, given by the energy divided by the frequency $(q E) /(2 \pi)$, is the instanton action

$$
\mathcal{S}_{\mathbf{k}_{\perp}}=2 S_{\mathbf{k}_{\perp}}=\pi \frac{m^{2}+\mathbf{k}_{\perp}^{2}}{q E} .
$$

The instanton action (24) can also be obtained in the time-dependent gauge $A_{\mu}=(0,0,0,-E t)$. Using the action $\mathcal{S}=\int L(t, \mathbf{x})$, Popov [10] obtained the same instanton action in the Coulomb gauge, and Affleck et al. [17] also obtained the same result in the mixed-gauge $A_{\mu}=-F_{\mu \nu} x_{\nu} / 2$. Therefore, the instanton action does not depend on the choice of gauge, which is just a matter of technical simplicity.

In a pure magnetic field $\mathbf{B}=B \mathbf{e}_{z}$ along the $\mathrm{z}$-direction, $\mathbf{A}=(0, B x, 0)$, and the component field equation of the Klein-Gordon or Dirac equation has a solution of the form

$$
\Phi=e^{i\left(k_{y} y+k_{z} z-\omega t\right)} \Phi_{\sigma_{ \pm} \omega k_{y} k_{z}}(x),
$$

which leads to the mode equation

$$
\left[-\partial_{x}^{2}+\left(q B x+k_{y}\right)^{2}+m^{2}+k_{z}^{2}-2 q B \sigma_{ \pm}-\omega^{2}\right] \Phi_{\sigma_{ \pm} \omega k_{y} k_{z}}(x)=0 .
$$

Here $\sigma_{ \pm}$is the spin projection: $\sigma=0$ for the scalar and $\sigma_{ \pm}= \pm 1 / 2$ for the Dirac spinor. The mode equation (26) has bound states given by harmonic wave functions with the energy spectrum [18]

$$
\omega^{2}=m^{2}+k_{z}^{2}+q B\left(2 j+1-2 \sigma_{ \pm}\right), \quad(j=0,1, \cdots) .
$$

The discrete spectrum due to the magnetic field is the Landau levels for charged particles. Note that all the Landau levels are non-degenerate for the scalar particles, whereas all the states of the Dirac spinor are doubly degenerate, $j, \sigma_{+}=1 / 2$ and $j-1, \sigma_{-}=-1 / 2$, except for the unique lowest Landau level, $j=0, \sigma_{+}=1 / 2$.

Now we apply the formulae [3] for pair production based on the instanton calculation to the static uniform electric and magnetic fields. For the electric and magnetic fields parallel to each other along the z-direction, the 4-potential is given by

$$
A_{\mu}=(-E z, 0, B x, 0)
$$

The component field equation,

$$
\left[\left(\partial_{t}-i q E z\right)^{2}-\partial_{x}^{2}-\left(\partial_{y}+i q B x\right)^{2}-\partial_{z}^{2}+m^{2}-2 q B \sigma_{ \pm}\right] \Phi_{\sigma_{ \pm}}=0,
$$

has the solution of the form

$$
\Phi_{\sigma_{ \pm}}=e^{i\left(k_{y} y-\omega t\right)} \Phi_{\sigma_{ \pm} \omega k_{y}}(x, z)
$$

Then the above equation becomes

$$
\left[\left\{-\partial_{x}^{2}+\left(q B x+k_{y}\right)^{2}\right\}+\left\{-\partial_{z}^{2}-(\omega+q E z)^{2}\right\}+m^{2}-2 q B \sigma_{ \pm}\right] \Phi_{\sigma_{ \pm} \omega k_{y}}(x, z)=0 .
$$

The first parenthesis has the harmonic wave functions as eigenfunctions, so the remaining equation becomes

$$
\left[-\partial_{z}^{2}-(\omega+q E z)^{2}+m^{2}+q B\left(2 j+1-2 \sigma_{ \pm}\right)\right] \Phi_{\sigma_{ \pm} \omega k_{y} j}(z)=0, \quad(j=0,1, \cdots,) .
$$

Therefore, the charged particles, exactly described by the inverted harmonic potential, have, as the instanton action for tunneling states,

$$
2 S_{\sigma_{ \pm} j}=\pi \frac{m^{2}+q B\left(2 j+1-2 \sigma_{ \pm}\right)}{q E} .
$$

The main result of Ref. [3], when converted from the imaginary part of the effective action to the pair-production, is that the expected number of pairs produced per mode in a static electric field is given by

$$
N_{ \pm}= \pm\left(e^{ \pm w}-1\right)=e^{-2 S}
$$

where the upper (lower) sign is for bosons (fermions) and $S$ is the instanton action for the corresponding mode, here given in Eq. [33). We then obtain the pair-production rate [19] for scalar particles as

$$
\mathcal{N}_{\text {scalar }}^{(1)}(E, B, m)=\frac{(q E)(q B)}{(2 \pi)^{2}} \sum_{j=0}^{\infty} \exp \left(-\pi \frac{m^{2}+q B(2 j+1)}{q E}\right)
$$


and for fermions as

$$
\mathcal{N}_{\text {spinor }}^{(1)}(E, B, m)=\frac{(q E)(q B)}{(2 \pi)^{2}} \sum_{j=0}^{\infty} \sum_{\sigma_{ \pm}= \pm 1 / 2} \exp \left(-\pi \frac{m^{2}+q B\left(2 j+1-2 \sigma_{ \pm}\right)}{q E}\right) .
$$

Here $(q E) /(2 \pi)$ is the (number) density of states (per momentum) from the $\omega$-integration, and $(q B) /(2 \pi)$ is that available for each Landau level. When we sum the geometric series of Eqs. (35) and (36), we readily get the standard results of Eqs. (3) and (4) respectively.

It should be noted that the pair-production rate does not depend on the renormalization scheme, since all divergence and renormalizability is contained in $\operatorname{Re} \mathcal{L}[2,14]$.

\section{INHOMOGENEOUS FIELD}

We now extend the analysis to inhomogeneous electric fields together with a constant magnetic field. As inhomogeneous electric fields we consider a localized constant electric field in the region $|z| \leq L$ and an electric field obtained from the Sauter potential $A_{0}(z)=-E L \tanh (z / L)$ for $q E L \gg m$ and $m L \gg 1$. In both cases, the electric field extends effectively a distance $2 L$ in the $z$-direction. For an electric field localized in the $z$-direction, pairs are produced only when $\omega-q A_{0}(+\infty) \geq m$ and $\omega-q A_{0}(-\infty) \leq-m$. Also the instanton actions exist when the Landau levels are limited to

$$
q B\left(2 j_{\max }+1\right)=\min \left\{\left(\omega-q A_{0}(+\infty)\right)^{2}-m^{2},\left(\omega-q A_{0}(-\infty)\right)^{2}-m^{2}\right\} .
$$

The pair-production rate per area and per time (using an overbar to distinguish from rates per volume per time) now takes the form, for scalar QED,

$$
\overline{\mathcal{N}}_{\text {scalar }}^{(1)}=\frac{(q B)}{(2 \pi)^{2}} \int_{q A_{0}(+\infty)+m}^{q A_{0}(-\infty)-m} d \omega \sum_{j=0}^{j_{\max }} e^{-2 S_{j}}
$$

and for spinor QED

$$
\overline{\mathcal{N}}_{\mathrm{spinor}}^{(1)}=\frac{(q B)}{(2 \pi)^{2}} \int_{q A_{0}(+\infty)+m}^{q A_{0}(-\infty)-m} d \omega \sum_{j=0}^{j_{\max }} \sum_{\sigma_{ \pm}= \pm 1 / 2} e^{-2 S_{\sigma_{ \pm} j}},
$$

where $(q B) /(2 \pi)$ is the number of state for Landau levels and another factor $1 /(2 \pi)$ is from the $\omega$ integration. The $\omega$ integration yields the potential energy difference

$$
V=q A_{0}(-\infty)-q A_{0}(+\infty)=2 q E L,
$$

where $L$ would extend to infinity for the homogeneous electric field. Here the instanton actions are determined by

$$
S_{\sigma_{ \pm} j}=\sum_{k=0}^{\infty} S_{\sigma_{ \pm} j}^{(2 k)}
$$

where the dominant (0-loop or classical) contribution comes from

$$
S_{\sigma_{ \pm} j}^{(0)}=\int_{z_{-}}^{z_{+}} d z \sqrt{m^{2}+q B\left(2 j+1-2 \sigma_{ \pm}\right)-\left(\omega-q A_{0}(z)\right)^{2}}
$$

where $z_{ \pm}$are turning points of the integral and $\sigma=0$ for scalar and $\sigma_{ \pm}= \pm 1 / 2$ for spinor QED.

In the first case the electric field is confined to a finite region of length $2 L$. The potential is given by

$$
A_{\mu}=(-E z, 0, B x, 0), \quad|z| \leq L .
$$

The instanton actions are given by

$$
2 S_{j}=\pi \frac{m^{2}+q B(2 j+1)}{q E}
$$


for $j$ smaller or equal to the highest Landau level

$$
j_{\max }=\frac{1}{2 q B}\left[\left(\frac{V}{2}-|\omega|\right)^{2}-m^{2}\right]-\frac{1}{2} .
$$

Finally, after the $\omega$ integration, we obtain the pair-production rate per area and per time for scalar QED

$$
\begin{aligned}
\overline{\mathcal{N}}_{\text {scalar }}^{(1)}= & \frac{(q E)(q B)}{2(2 \pi)^{2}} \operatorname{csch}\left(\frac{\pi B}{E}\right) \exp \left(-\frac{\pi m^{2}}{q E}\right) \\
& \times(2 L)\left[1-\frac{2 m}{V}+\frac{2 m}{V} \exp \left(-\frac{\pi B}{E}\right) \int_{0}^{\sqrt{(V / 2 m)^{2}-1}} d x \frac{x e^{-\frac{\pi m^{2}}{q E} x^{2}}}{\sqrt{1+x^{2}}}\right],
\end{aligned}
$$

and that for spinor QED

$$
\begin{aligned}
\overline{\mathcal{N}}_{\text {spinor }}^{(1)}= & \frac{(q E)(q B)}{(2 \pi)^{2}} \operatorname{coth}\left(\frac{\pi B}{E}\right) \exp \left(-\frac{\pi m^{2}}{q E}\right) \\
& \times(2 L)\left[1-\frac{2 m}{V}+\frac{2 m}{V} \exp \left(-\frac{\pi B}{E}\right) \int_{0}^{\sqrt{(V / 2 m)^{2}-1}} d x \frac{x e^{-\frac{\pi m^{2}}{q E} x^{2}}}{\sqrt{1+x^{2}}}\right] .
\end{aligned}
$$

We note that the first factor is the pair-production rate per volume for the constant electric field in Sec. II, and the localization of the electric field gives the factor on the second line, which is roughly $2 L$ for $V \gg 2 m$. In the large $L$ limit, after dividing by $2 L$ to convert from a rate per area to a rate per volume, we recover Eqs. (3i) and (4), since $m / V \rightarrow 0$.

Next, we turn to the slowly varying electric field $E(z)=E \operatorname{sech}^{2}(z / L)$ given by the Sauter potential

$$
A_{0}(z)=-E L \tanh \left(\frac{z}{L}\right) \text {, }
$$

for $q E L \gg m$ and $m L \gg 1$. The highest Landau level is the same as Eq. (45). However, the instanton actions are given by

$$
2 S_{\sigma_{ \pm} j}=\frac{\pi m^{2} \alpha}{q E}+\frac{\pi B \beta}{E}\left(2 j+1-2 \sigma_{ \pm}\right)+\mathcal{O}\left(\frac{j^{2}}{V^{2}}, \frac{1}{V^{4}}\right)
$$

where

$$
\begin{aligned}
& \alpha=1+4 \frac{\omega^{2}}{V^{2}}+\frac{m^{2}}{V^{2}}, \\
& \beta=1+4 \frac{\omega^{2}}{V^{2}}+2 \frac{m^{2}}{V^{2}} .
\end{aligned}
$$

The instanton actions are also obtained from Eq. (47) of Ref. [3] by replacing $\mathbf{k}_{\perp}^{2}$ by $q B\left(2 j+1-2 \sigma_{ \pm}\right)$. Then the pair-production rate per area and per time for scalar QED takes the form

$$
\begin{aligned}
\overline{\mathcal{N}}_{\text {scalar }}^{(1)}= & \frac{(q B)}{2(2 \pi)^{2}} \int_{-\left(\frac{V}{2}-m\right)}^{\left(\frac{V}{2}-m\right)} d \omega \operatorname{csch}\left(\frac{\pi B \beta}{E}\right) \exp \left(-\frac{\pi m^{2} \alpha}{q E}\right) \\
& \times\left[1-\exp \left(-\frac{\pi B \beta}{E}\right) \exp \left\{-\frac{\pi \beta}{q E}\left(\left(\frac{V}{2}-|\omega|\right)^{2}-m^{2}\right)\right\}\right] .
\end{aligned}
$$

The spinor case is obtained by replacing $\operatorname{csch}(\pi B \beta / E)$ by $2 \operatorname{coth}(\pi B \beta / E)$, the 2 being for two spins. In the $L=\infty$ limit, with $\int d \omega=V=2 q E L=\infty$, we recover the standard result (33) and (4) when we divide by $2 L$ to get the rate per volume instead of the rate per area. In fact, we can see that the final factor of the right hand side of Eq. (51), the part inside the square brackets, is nearly one over most of the integral if $q E L^{2} \gg 1$, so that that factor can be dropped.

It is interesting to compare the pair-production rates per area given by the instanton method using just the classical action with the rate per area given by integrating the uniform-field rate per volume over the extent of the inhomogeneous field:

$$
\overline{\mathcal{N}}_{\mathrm{u}}=\int d z \mathcal{N}(E(z), B, m)
$$


Here the subscript $u$ is for "uniform field".

For simplicity, let us take the case $B=0$, so the uniform-field rate per volume is the same for bosons and for fermions (per spin state) and in the Sauter electric field $E(z)=\operatorname{Esech}^{2}(z / L)$ would be

$$
\mathcal{N}=\frac{(q E)^{2}}{(2 \pi)^{3}} \operatorname{sech}^{4}\left(\frac{z}{L}\right) \exp \left[-\frac{\pi m^{2}}{q E} \cosh ^{2}\left(\frac{z}{L}\right)\right] .
$$

If we let

$$
\delta \equiv \frac{q E}{\pi m^{2}}
$$

(where this $E$ is the maximum value of the Sauter electric field), and if we let $x=\sinh (z / L)$, we get

$$
\overline{\mathcal{N}}_{\mathrm{u}}=\frac{(q E)^{2} L}{(2 \pi)^{3}} e^{-\frac{1}{\delta}} \int_{-\infty}^{\infty} \frac{d x}{\left(1+x^{2}\right)^{5 / 2}} e^{-\frac{x^{2}}{\delta}} .
$$

Analogously, if in Eq. (51) we set $x=2 \omega / V$ and

$$
\epsilon \equiv \frac{2 m}{V}=\frac{m}{q E L} \ll 1,
$$

then assuming $\delta \epsilon^{2}=1 /\left(\pi q E L^{2}\right) \ll 1$ so that the final factor in Eq. (51) may be dropped, that equation gives

$$
\overline{\mathcal{N}}_{\mathrm{i}} \approx \frac{(q E)^{2} L}{(2 \pi)^{3}} e^{-\frac{1}{\delta}} \int_{-1}^{1} \frac{d x}{1+x^{2}} e^{-\frac{x^{2}}{\delta}}
$$

Here the subscript i stands for "instanton method", here using just the 0-loop or classical action.

If we now have a maximum electric field $E$ far below the critical value $m^{2} / q$, so $\delta \ll 1$, then

$$
\overline{\mathcal{N}}_{\mathrm{u}} \approx e^{\frac{\epsilon^{2}}{4 \delta}} \overline{\mathcal{N}}_{\mathrm{i}} \approx \frac{(q E)^{2} L}{(2 \pi)^{3}} \sqrt{\pi \delta} e^{-\frac{1}{\delta}}=\frac{(q E)^{5 / 2} L}{(2 \pi)^{3} m} e^{-\frac{1}{\delta}}
$$

The two estimates for the rate agree if

$$
\frac{\epsilon^{2}}{4 \delta}=\frac{\pi m^{4}}{4(q E)^{3} L^{2}}=\frac{\pi m^{4}}{q E V^{2}}=\frac{2 \pi m^{4} L}{V^{3}} \ll 1,
$$

but they do not agree if this quantity is not small.

On the other hand, if the maximum electric field $E$ is far above the critical value $m^{2} / q$, so $\delta \gg 1$, then necessarily $\epsilon^{2} / \delta \ll 1$, and we get

$$
\overline{\mathcal{N}}_{\mathrm{u}} \approx \frac{8}{3 \pi} \overline{\mathcal{N}}_{\mathrm{i}} \approx \frac{(q E)^{2} L}{6 \pi^{3}} \sqrt{\pi \delta}
$$

Thus in this case the uniform-field approximation gives roughly $85 \%$ of the 0-loop instanton-method approximation using only the leading term for the actions.

It is also of interest to compare these results with the exact results for the Sauter electric field. Nikishov [13] has given the expected number of pairs per mode, but one must integrate over the modes to get the pair-production rate per area. Here we shall restrict ourselves to spinor case, as it is actually simpler. Then if one defines

$$
Z \equiv 2 \pi q E L^{2}=\frac{2}{\delta \epsilon^{2}}
$$

the pair-production rate per area for spinors (with two spin states) can be shown after some algebra to be

$$
\overline{\mathcal{N}}_{\text {spinor }}=2 \frac{(q E L)^{3}}{(2 \pi)^{2}} \int \frac{\cosh Z y-\cosh Z x}{\cosh Z-\cosh Z x}\left(y^{2}-x^{2}\right) d x d y
$$

where in this integral we have the restrictions

$$
\begin{array}{r}
-1 \leq-y \leq x \leq y \leq 1 \\
\left(1-x^{2}\right)\left(1-y^{2}\right) \geq\left(\frac{2 m}{V}\right)^{2}=\epsilon^{2} .
\end{array}
$$


This result is exact (to 1-loop in the quantum field theory) for any $Z$ and and $\epsilon \leq 1$, pair-production being energetically impossible if $\epsilon>1$.

One can now show that if $\delta \ll \sqrt{1-\epsilon^{2}}$, a good approximation to this integral expression for any $\epsilon \leq 1$ is

$$
\overline{\mathcal{N}}_{\text {spinor }} \approx \frac{(q E)^{2} L}{4 \pi^{3}} \sqrt{\pi \delta}\left(1-\epsilon^{2}\right)^{5 / 4} e^{-Z\left(1-\sqrt{1-\epsilon^{2}}\right)} .
$$

For the particular subcase considered above, $\epsilon \ll 1$ and $\delta \ll 1$, so long as $\delta \ll \epsilon^{4}$ one gets agreement with the instanton-method approximation given in Eq. (58) (after multiplying that expression by the 2 spin states of the spinor).

In particular, for $\delta^{2} \ll \epsilon^{4} \ll \delta$, (where the first subdominant term in the expansion of the exponent in (64) is large, but not the second subdominant term), the approximation (64) to the exact answer (62) agrees with the 0-loop instanton-method approximation (58) but not with the uniform-field approximation there, which is larger by the factor $e^{\epsilon^{2} /(4 \delta)}$. However, for $\delta \ll \epsilon^{4}$, then even the second subdominant term in the expansion of the exponent in (64) is large in comparison with unity, so then the exact result is significantly different from both the uniform-field approximation and the 0-loop instanton-method approximation (58), at least if (64) is a good approximation to the exact result to all orders in $\epsilon$ when $\delta \ll \sqrt{1-\epsilon^{2}}$. However, we do obtain precisely (64) by properly calculating the 0 -loop instanton action and making a gaussian approximation for the integral over $\omega$ and $\mathbf{k}_{\perp}$.

In the case in which $\epsilon^{2} \ll \delta \ll 1$, then both approximations agree closely with the exact result. When we retain the assumption that $\epsilon \ll 1$ but go to the opposite limit of high maximum electric field strength, so $\delta \gg 1$, then the exact integral expression (62) reduces to the uniform-field approximation of Eq. (60) (after that is multiplied by the factor of 2), rather than to the 0-loop instanton-method approximation also given there. That is, the 0-loop instanton-method approximation gives a result that is too large by a factor of $3 \pi / 8 \approx 1.178$. This is what we might expect, since the potential is changing rapidly so the 0-loop instanton approximation, using just the lowest-order (classical) action as we have done here, would not necessarily be expected to be good in this limit. And yet it is encouraging that it is only off by less than $20 \%$ for the pair-production rate.

While this paper was being revised, we have become aware of some related papers that have recently appeared in the literature [22, 23, 24, 25, 26, 27, 28] which use the worldline method. For a static electric field in the $z$-direction that depends only on $z$, the single-worldline instanton action $S_{0}$ is the same as the minimum value of our 0-loop or classical instanton action $2 S_{\mathbf{k}_{\perp}}^{(0)}(\omega)$ as a function of the transverse momentum $\mathbf{k}_{\perp}$ and the frequency $\omega$. (The minimum is always at $\mathbf{k}_{\perp}=0$, and in the special cases in which scalar potential is an antisymmetric function of $z$, $A_{0}(z)=-A_{0}(-z)$, as in, e.g., the Sauter potential, the minimum is also at $\omega=0$.)

Dunne, Gies, Schubert, and Wang have now worked out the subleading prefactor contribution [28] using the worldline instanton method, which appears to agree with the gaussian approximation for the integrals over $\omega$ and $\mathbf{k}_{\perp}$ in our approach using $2 S_{\mathbf{k}_{\perp}}^{(0)}(\omega)$. For the Sauter potential, both instanton methods give exactly the same expression as Eq. (64) which we obtained as an approximation to the exact double-integral Eq. (62) from Nikishov [13].

\section{CONCLUSION}

In conclusion, using the instanton method in the Coulomb (space-dependent) gauge for constant electric and magnetic fields, we have obtained new forms (35) and (36) for the pair-production rate in scalar QED and spinor QED. These formulae agree exactly with the well-known results. Finally, we suggested a generalization of these formulae to a constant magnetic field and an inhomogeneous electric field, and compared the results to another method and to the exact answer for the Sauter electric potential.

\section{Acknowledgments}

The authors thank G. Dunne, H. Gies, A. I. Nikishov, S. J. Rey, and C. Schubert for useful discussions, and S.P.K. expresses his appreciation for the warm hospitality of the Theoretical Physics Institute, University of Alberta. The work of S.P.K. was supported by the Korea Science and Engineering Foundation under Grant Nos. 1999-2-112-003-5 and R01-2005-000-10404-0, and the work of D.N.P. by the Natural Sciences and Engineering Research Council of Canada. 


\section{APPENDIX A: TUNNELING AND REFLECTION PROBABILITY}

In the context of nonrelativistic quantum theory, we derive a formula for the tunneling and reflection coefficients for bosons under a general potential barrier.

Let the wave equation take the form

$$
y^{\prime \prime}(z)+Q(z) y(z)=0, \quad Q=E-V(z),
$$

and have three different regions:

$$
\begin{cases}I=\left(-\infty, z_{1}\right), & Q>0 \\ I I=\left(z_{1}, z_{2}\right), & Q<0 \\ I I I=\left(z_{2}, \infty\right), & Q>0\end{cases}
$$

Here $z_{1}$ and $z_{2}$ are two turning points. We assume that an incoming wave function from $-\infty$ in region (I) is partially reflected back to $-\infty$ and partially tunnels through the barrier in region (II) toward $\infty$ in region (III). Then the wave function in region (I) is given by

$$
y=y_{I}+B y_{I}^{*},
$$

where $y_{I}$ has the incoming flux toward the barrier, and in region (III)

$$
y=C y_{I I I}
$$

(assuming an outgoing flux there, which actually corresponds to an incoming group velocity for the relativistic waves of our paper). Here the incoming flux is normalized to unity. The flux conservation for equal momenta at $z= \pm \infty$ leads to the relation

$$
|B|^{2}+|C|^{2}=1
$$

Then we may find a wave function of the form [20]

$$
y_{I}=\exp \left[\sum_{k=0}^{\infty} S^{(2 k)}\right]
$$

with $\operatorname{Im}\left(d S_{0} / d z\right)>0$ to conform with the direction of the incoming flux. The leading term in region (I) is given by

$$
S^{(0)}(z)=i \int_{-\infty}^{z} \sqrt{Q(z)} d z
$$

and in region (II)

$$
S^{(0)}=i \int_{z_{1}}^{z_{2}} \sqrt{Q(z)} d z
$$

is what may be called the 0-loop or classical action for the nonrelativistic problem.

We may analytically continue $y_{I}$ from region (I) to region (III) through region (II) along a semicircle starting from $z_{1}$ and ending at $z_{2}$ in the upper $z$-plane. Then $z \rightarrow e^{-i \pi} z=-z$, and the leading term, for instance, takes the form in region (III)

$$
S^{(0)}=-i \int_{\infty}^{z} \sqrt{Q(z)}+i \int_{z_{1}}^{z_{2}} \sqrt{Q(z)}+i\left(\int_{-\infty}^{z_{1}} \sqrt{Q(z)}+\int_{\infty}^{z_{2}} \sqrt{Q(z)}\right) .
$$

In Eq. A99, the first term in the right hand side has the incoming flux, hence is the leading term of $y_{I I I}^{*}$, and the second term is the instanton action, a real quantity, through the barrier, and the last term gives a phase factor. We may thus write the analytically continued wave function as

$$
y_{I} \rightarrow e^{S}\left(y_{I I I}^{*}+\alpha y_{I I I}\right) .
$$


So the wave function in region (I) is analytically continued in region (III) to

$$
y_{I}+B y_{I}^{*} \rightarrow e^{S}(\alpha+B) y_{I I I}+e^{S}\left(1+B \alpha^{*}\right) y_{I I I}^{*} .
$$

As there is no incoming wave function in region (III), we have

$$
e^{S}(\alpha+B)=C, \quad 1+\alpha^{*} B=0 .
$$

Solving Eq. A12 together with Eq. A5), we finally obtain the coefficients for tunneling and reflection

$$
|B|^{2}=\frac{1}{1+e^{-2 S}}, \quad|C|^{2}=\frac{1}{1+e^{2 S}},
$$

which confirms the result in Refs. [3] and [21].

[1] F. Sauter, Z. Phys. 69, 742 (1931); W. Heisenberg and H. Euler, Z. Physik 98, 714 (1936); V. Weisskopf, K. Dan. Vidensk. Selsk. Mat. Fys. Medd. 14, No. 6 (1936).

[2] J. Schwinger, Phys. Rev. 82, 664 (1951).

[3] S. P. Kim and D. N. Page, Phys. Rev. D 65, 105002 (2002).

[4] G. V. Dunne, "Heisenberg-Euler Effective Lagrangians: Basics and Extensions," From Fields to Strings: Circumnavigating Theoretical Physics, Vol I, M. Shifman et al (eds.), (World Scientific, Singapore, 2005), arXiv:hep-th/0406216.

[5] W. Greiner, B. Müller, and J. Rafelski, Quantum Electrodynamics of Strong Fields (Springer-Verlag, Berlin, 1985); W. Dittrich and H. Gies, Probing the Quantum Vacuum: Perturbative Effective Action Approach in Quantum Electrodynamics and its Applications (Springer-Verlag, Berlin, 2000).

[6] R. C. Duncan, "Physics in Ultra-strong Magnetic Fields," astro-ph/0002442 M. H. P. M. van Putten, Phys. Rev. Lett. 84, 3752 (2000), and references therein.

[7] D. L. Burke et al., Phys. Rev. Lett. 79, 1626 (1997); R. Alkofer, M. B. Hecht, C. D. Roberts, S. M. Schmidt, and D. V. Vinnik, ibid. 87, 193902 (2001); C. D. Roberts, S. M. Schmidt, and D. V. Vinnik, ibid. 89, 153901 (2002); B. Shen and M. Y. Yu, ibid. 89, 275004 (2002); for review and references, see A. Ringwald, "Boiling the Vacuum with an X-Ray Free Electron Laser," hep-ph/0304139

[8] F. V. Bunkin and I. I. Tugov, Sov. Phys. Dokl. 14, 678 (1970).

[9] J. K. Daugherty and I. Lerche, Phys. Rev. D 14, 340 (1976).

[10] V. S. Popov, Sov. Phys. JETP 34, 709 (1972); Sov. Phys. JETP 35, 659 (1972)

[11] C. Itzykson and J. B. Zuber, Quantum Field Theory (McGraw-Hill, New York, 1980).

[12] Y. M. Cho and D. G. Pak, Phys. Rev. Lett. 86, 1947 (2001).

[13] A. I. Nikishov, Sov. Phys. JETP 30, 660 (1970); Nucl. Phys. B21, 346 (1970).

[14] S. I. Kruglov, Ann. Phys. (N.Y.) 293, 228 (2001); Eur. Phys. J. C 22, 89 (2001); Int. Theor. Phys. 40, 515 (2001).

[15] E. Brezin and C. Itzykson, Phys. Rev. D 2, 1191 (1970); A. Casher, H. Neuberger, and S. Nussinov, Phys. Rev. D 20, 179 (1979); H. Neuberger, ibid. 20, 2936 (1979).

[16] M. Abramowitz and I. Stegun, Handbook of Mathematical Functions (Dover Pub., New York, 1964).

[17] I. K. Affleck, O. Avarez, and N. S. Manton, Nucl. Phys. B197, 509 (1982).

[18] W. Greiner and J. Reinhardt, Quantum Electrodynamics (Springer-Verlag, Berlin, 1992) p 280, pp $286-287$.

[19] There are different terms for $w=2 \operatorname{Im} \mathcal{L}$, which is not exactly the same as the pair-production rate. But since it agrees to lowest order when small, Schwinger [2] used the term 'pair-production probability' for $w$, and Itzykson and Zuber 11] used 'pair-production rate'. Nikishov uses 'pair-production' only for the quantities of Eqs. (3) and (4). For a single mode, the relation is given by Eq. (34). In fact, $w$ describes the vacuum-to-vacuum persistence or the decay rate of the vacuum.

[20] C. M. Bender, K. Olaussen, and P. S. Wang, Phys. Rev. D 16, 1740 (1977); C. M. Bender and S. A. Orszag, Advanced Mathematical Methods for Scientists and Engineers I (Springer, New York, 1999).

[21] N. Fröman and P. O. Fröman, Nucl. Phys. A147, 606 (1970); Phase-Integral Method (Springer, New York, 1996).

[22] H. Gies and K. Langfeld, Nucl. Phys. B613, 353 (2001); Int. J. Mod. Phys. A 17, 966 (2002).

[23] K. Langfeld, L. Moyaerts, and H. Gies, Nucl. Phys. B646, 158 (2002); J. High Energy Phys. 0306, 018 (2003).

[24] L. Moyaerts, K. Langfeld, and H. Gies, "Worldline Approach To The Casimir Effect," hep-th/0311168

[25] F. Bastianelli and C. Schubert, J. High Energy Phys. 0502, 069 (2005).

[26] H. Gies and K. Klingmüller, Phys. Rev. D 72, 065001 (2005).

[27] G. V. Dunne and C. Schubert, Phys. Rev. D 72, 105004 (2005).

[28] G. V. Dunne, Q.-H. Wang, H. Gies, and C. Schubert, "Worldline Instantons II: The Fluctuation Prefactor," hep-th/0602176 